\title{
Percutaneous angioplasty of the only patent renal artery - treatment of choice for end-stage heart failure
}

\author{
Jacek Kądziela, Paweł Tyczyński, Ilona Michałowska, Marek Konka, Bartosz Duda, Adam Witkowski \\ Institute of Cardiology, Warsaw, Poland
}

Postep Kardiol Inter 2013; 9, 1 (31): 83-85

DOI: $10.5114 /$ pwki.2013.34031

\begin{abstract}
The causes leading to the exacerbation of preexisting chronic heart failure can be various. Renal artery stenosis is an important cause of renal dysfunction, and can lead to worsening of heart failure and increased mortality. We present a 49-year old male patient after acute myocardial infarction, heart failure with left ventricle ejection fraction of $20 \%$, permanent atrial flutter, peripheral vascular disease, chronic kidney disease and arterial hypertension. In 2008 the patient underwent stenting of the left renal artery. At that time there was only discrete stenosis of the right renal artery. Two years later the patient presented with exacerbation of bi-ventricular chronic heart failure and renal failure. The echocardiography revealed dramatically reduced systolic function of both ventricles. As no response to intravenous diuretics was achieved, the patient underwent several courses of hemodialysis. Before considering the patient as a potential candidate for orthotopic heart transplantation, color Doppler ultrasound showed the occlusion of the left renal artery and significant stenosis of the right renal artery, which was stented. After the procedure spectacular clinical improvement was observed. The results of two randomized trials, ASTRAL and STAR, were discouraging for invasive treatment of renal artery stenosis. Still, serious doubts regarding the methodology of the studies were raised. Renal artery stenting may be the treatment of choice in highly selected patients and lead to clinical improvement.
\end{abstract}

Key words: renal artery stenting, end-stage heart failure.

\section{Case report}

We present a 49-year-old male patient after acute myocardial infarction (MI) of the anterior wall treated with primary angioplasty of the left anterior descending artery with implantation of a bare metal stent (BMS) in 2008, postMI NYHA III HF with left ventricle ejection fraction (LVEF) of $20 \%$, permanent atrial flutter (AFI), peripheral vascular disease, chronic kidney disease stage 3 and arterial hypertension. In 2008 the patient underwent renal artery stenting (RAS) for atherosclerotic renal artery stenosis (ARAS) of the left renal artery (LRA). At that time there was only discrete stenosis of the right renal artery (RRA) (Figure 1 A).

In November 2010 the patient presented with exacerbation of bi-ventricular chronic heart failure (HF) and renal failure associated with dyspnea at rest and massive fluid retention. The serum creatinine was $281 \mu \mathrm{mol} / \mathrm{l}$ and glomerular filtration rate (GFR) was $21 \mathrm{ml} / \mathrm{min} / 1.73 \mathrm{~m}^{2}$. The ECG demonstrated AFl, and Q waves in inferior and precordial leads. The echocardiography revealed enlargement of all cardiac chambers, dramatically reduced systolic function of both ventricles with LVEF of $11 \%$, elevated systolic right ventricle (RV) pressure up to $50 \mathrm{~mm} \mathrm{Hg}$ and significant tricuspid regurgitation (TR).

As no response to intravenous diuretics was achieved, the patient underwent several courses of hemodialysis, followed by oral diuretics with moderate clinical response. Additionally, implantation of a dual-chamber ICD was performed for intermittent advanced atrioventricular block and as a primary prevention of sudden cardiac death. Considering the tachyarrhythmia as a possible cause aggravating the cardiac output, successful radiofrequency ablation of the AFI substrate was done. In the course of the following few weeks the patient was in a stable condition, but with lower limbs edema and moderate ascites. Before considering the patient as a potential candidate for orthotopic heart transplantation (OHT) for end-stage HF, color Doppler ultrasound (CDU) of the renal arteries was done to screen for

\section{Corresponding author:}

Paweł Tyczyński MD, PhD, Department of Interventional Cardiology and Angiology, Institute of Cardiology, 42 Alpejska St, 04-628 Warsaw, Poland, tel.: +48 2234342 72, e-mail: medykpol@wp.pl

Received: 17.10.2012, accepted: 8.11.2012. 
possible causes of persistent fluid overload. This showed significant stenosis of the RRA. The proximal segment of the LRA was not visualized, but intrarenal flow parameters (e.g. resistive index) strongly suggested tight stenosis. Therefore in March 2011, aortography was performed via the left brachial artery, which revealed tight RRA ostial stenosis and occlusion of the abdominal aorta above the LRA origin resulting in its occlusion (Figures $1 \mathrm{~B}, \mathrm{C}$ ). Direct implantation of a BMS (Herculink Elite 5.5/15 mm at 14 atm) was performed with a good angiographic result (Figure $1 \mathrm{D}$ ). After the procedure, spectacular clinical improvement with polyuria of more than 10 I within 2 days was observed, followed by decrease of the ascites and edema of lower limbs. The patient was discharged with medical treatment including acetylsalicylic acid, clopidogrel, statin, $\beta$-blocker, angiotensin converting enzyme (ACE) inhibitor, digoxin, eplerenone, hydrochlorothiazide and furosemide reduced by half. Of note, as no suspicion of significant stenosis of the only patent renal artery was raised before, the patient has been permanently treated with an ACE inhibitor.

Control CDU 3 months after RAS showed patent RRA without restenosis. The control echocardiography confirmed
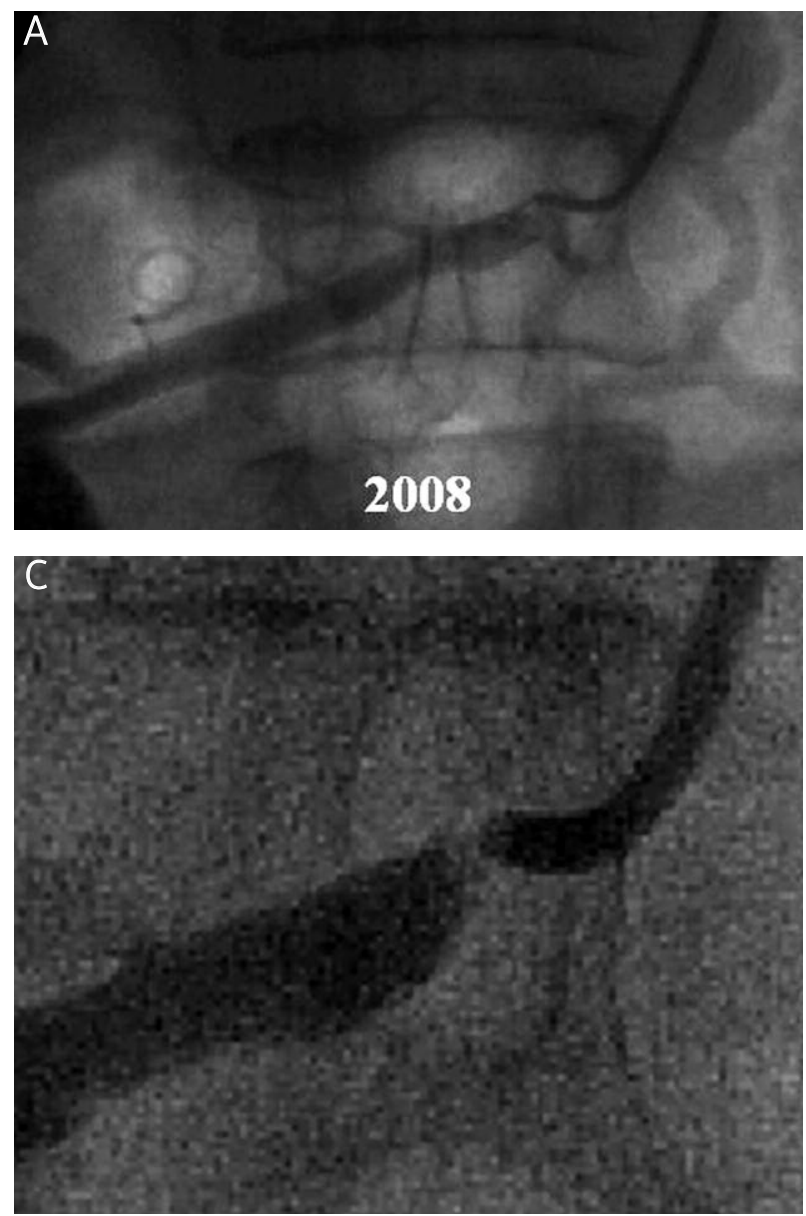

significant LV dysfunction, but with modest improvement (LVEF 25\%) and moderate TR.

NT-pro-BNP decreased from $18873 \mathrm{pg} / \mathrm{ml}$ in March 2011 to $3285 \mathrm{pg} / \mathrm{ml}$ (normal range: $0-125 \mathrm{pg} / \mathrm{ml}$ ). Creatinine level decreased to $153 \mu \mathrm{mol} / /$ with improvement of GFR to $42 \mathrm{ml} / \mathrm{min} / 1.73 \mathrm{~m}^{2}$. The patient was in NYHA II functional class and is no longer considered as a candidate for OHT.

\section{Discussion}

The progression of ARAS despite satisfactory lipid lowering treatment may be insidious. In patients with no satisfactory response to $\mathrm{HF}$ treatment all possibly reversible causes should be meticulously considered, especially in patients with a previous history of RAS of the LRA. As significant renal artery stenosis of the solitary functioning kidney was not known, the patient was permanently treated with an ACE inhibitor until the diagnosis. This obviously did not help to stabilize the renal function and could even lead to its deterioration and subsequently exacerbate underlying HF. Common percutaneous treatment of ARAS is questioned and the benefits associated with PRA versus medical therapy alone remain unproven. Still, renal artery revascular-
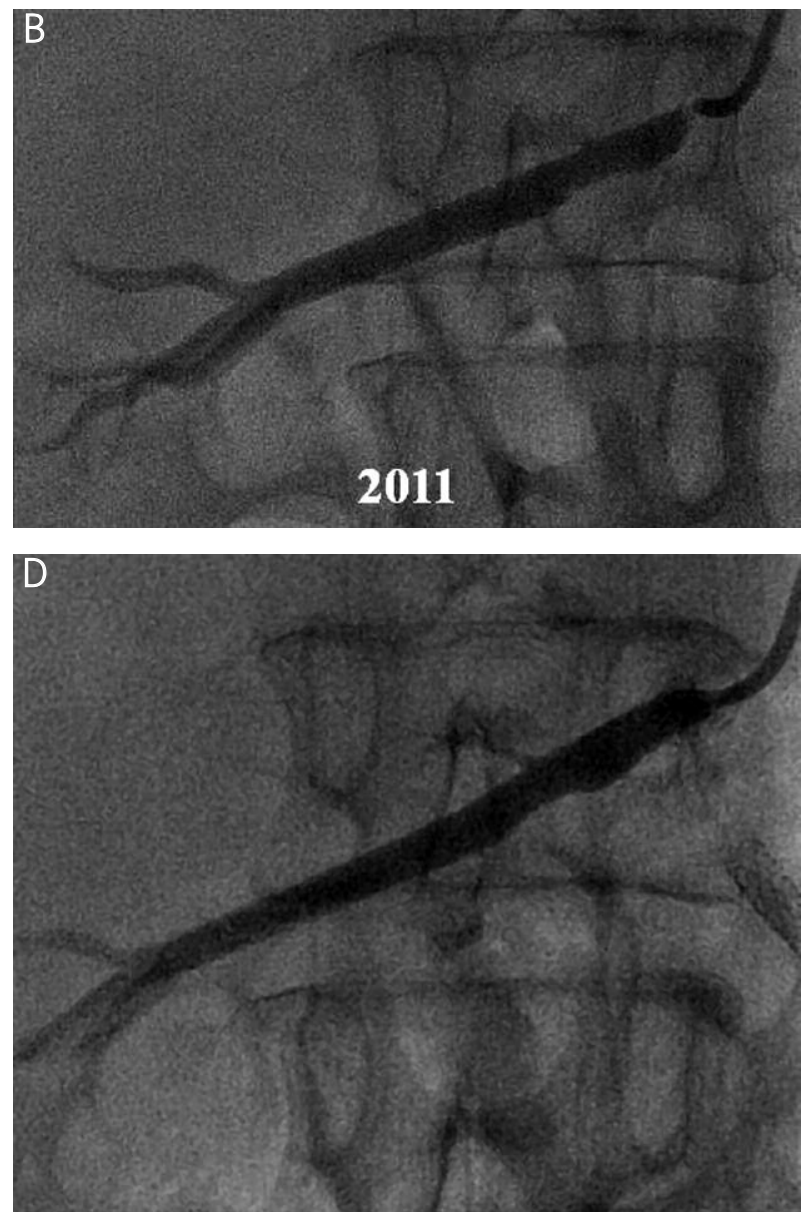

Fig. 1. A - Discrete stenosis of the right renal artery (RRA) in 2008. B - Tight ostial stenosis of the RRA in 2011. C - Magnification of image "B". D - Angiographic result after stenting 
ization for significant stenosis may represent the only treatment option for HF patients unresponsive to medical management. The results of two randomized trials, ASTRAL [1] and STAR [2], comparing RAS vs. medical therapy in patients with significant ARAS, were discouraging for the RAS strategy. Still, serious doubts regarding the methodology of the studies were raised [3].

Retrospective studies assessing safety and efficacy of RAS of solitary functioning kidney showed some benefits and acceptable complication rates [4].

When obstructions of the femoro-iliac arteries preclude groin access, the transbrachial access can be considered, and this route was the only option in our patient.

Follow-up observation of our patient showed significant preservation of kidney function and modest improvement of heart function.

\section{References}

1. ASTRAL Investigators; Wheatley K, Ives N, Gray R, et al. Revascularization versus medical therapy for renal-artery stenosis. $N$ Engl J Med 2009; 361: 1953-1962.

2. Bax L, Woittiez AJ, Kouwenberg HJ, et al. Stent placement in patients with atherosclerotic renal artery stenosis and impaired renal function: a randomized trial. Ann Intern Med 2009; 150: 840-848.

3. Henry M, Benjelloun A, Henry I, et al. Renal angioplasty and stenting: is it still indicated after ASTRAL and STAR studies? J Cardiovasc Surg 2010; 51: 701-720.

4. Tan J, Filobbos R, Raghunathan G, et al. Efficacy of renal artery angioplasty and stenting in a solitary functioning kidney. Nephrol Dial Transplant 2007; 22: 1916-1919. 